Relations industrielles

Industrial Relations

\title{
Le contrôle des monopoles
}

\section{Gérald Marion}

Volume 15, numéro 1, janvier 1960

URI : https://id.erudit.org/iderudit/1022074ar

DOI : https://doi.org/10.7202/1022074ar

Aller au sommaire du numéro

\section{Éditeur(s)}

Département des relations industrielles de l’Université Laval

\section{ISSN}

0034-379X (imprimé)

1703-8138 (numérique)

Découvrir la revue

\section{Citer ce document}

Marion, G. (1960). Le contrôle des monopoles. Relations industrielles / Industrial Relations, 15(1), 118-126. https://doi.org/10.7202/1022074ar
Résumé de l'article

Quelques commentaires en marge du bill C-59
Tous droits réservés (C Département des relations industrielles de l’Université Laval, 1960
Ce document est protégé par la loi sur le droit d'auteur. L’utilisation des services d'Érudit (y compris la reproduction) est assujettie à sa politique d'utilisation que vous pouvez consulter en ligne.

https://apropos.erudit.org/fr/usagers/politique-dutilisation/ 
Elle en déduit que ce fait devrait généralement être reconnu et admis par l'industrie, et que s'il ne l'était pas, il faudrait que l'Etat intervienne. Ce langage me paraît un peu étonnant quand on l'accouple aux compliments prodigués à l'industrie dans le rapport.

D’autres conclusions sont beaucoup mieux amenées. Par exemple, le rapport suggère l'établissement d'un conseil permanent qui surveillerait prix, productivité et profits dans l'industrie alimentaire et présenterait un rapport annuel. Il recommande aussi que, si les bons-primes et timbres-primes survivent, le client ait le droit de se faire rembourser en espèces par le détaillant, de façon à obtenir une ristourne. Il demande l'établissement d'un office fédéral destiné à renseigner le consommateur sur les lois et les services qui le protègent. En vue d'aider le petit épicier, les commissaires désirent que l'on recueille et mette à sa disposition des renseignements détaillés sur l'administration des petites entreprises. Pour aider les fermiers, il faudrait adopter une loi fédérale prévoyant la constitution en compagnies des coopératives. Enfin, le rapport insiste sur le besoin de recueillir plus de statistiques.

Somme toute, personne n'est oublié, pour son bonheur ou pour son malheur.

En conclusion générale, je dirai que la Commission Stewart a fait un gros travail de déblaiement dans une question complexe et que les plus intéressantes recommandations sont justement celles qui visent à mener à bonne fin une oeuvre si bien commencée.

\section{Le contrôle des monopoles}

Quelques commentaires en marge du bill C-59

\section{Gérald Marion}

L'année dernière, le gouvernement fédéral avait distribué à la Chambre des Communes le bill C-59 dont l'objet est d'apporter des changements substantiels à la législation anti-trust existante. A la suite de protestations, le bill fut retiré avant qu'il ait fait l'objet de débats parlementaires. On peut s'attendre, cependant, qu'au cours de la prochaine session, le gouvernement revienne à la charge avec un nouveau projet. C'est pourquoi il n'est pas inutile de faire une analyse de l'ancien projet de loi.

\section{LA LOI ACTUELLE}

Selon la présente loi des enquêtes sur les coalitions et son interprétation dans la jurisprudence, toute entente commerciale ou industrielle est illégale parce qu'elle est une menace à l'intérêt public. Le lien qui 
relie les ententes industrielles ou commerciales à la menace à l'intérêt public, c'est le pouvoir obtenu par certains groupes particuliers, grâce à la disparition de la concurrence. Comme l'a dit l'honorable juge Migneault, «... Le détriment causé au public par l'empêchement ou la suppression de la concurrence, est ce qui fait tomber un accord ou une coalition sous la proscription de l'article 498 C.P.C. ». ${ }^{1}$ La présente loi a donc pour but de sauvegarder l'intérêt public, la mesure de la sauvegarde de cet intérêt étant la concurrence ou tout au moins l'absence de coalition entre les fabricants ou les distributeurs d'une marchandise. Le principe dirigeant en ce qui concerne la règlementation des monopoles est basé sur le fait qu'aucune association privée ne peut déterminer arbitrairement les conditions industrielles et commerciales qui doivent prévaloir dans un secteur de l'économie. Cependant, on peut se demander s'il est possible d'après la présente loi anti-monopole qu'une entente ayant pour but de restreindre la concurrence dans le commerce soit permise si elle n'est pas une menace directe à l'intérêt public. Lors de la présentation de la loi contre les coalitions de 1923, au Parlement canadien, le premier ministre d'alors avait fait certaines déclarations selon lesquelles il pouvait y avoir de bonnes et de mauvaises ententes, seules ces dernières étant néfastes à l'intérêt public et ainsi condamnables. ${ }^{2}$ Cependant, par suite de l'interprétation de la loi anticoalition dans la jurisprudence des tribunaux, cette distinction entre les bonnes et les mauvaises ententes paraît superflue. En effet, quel que soit le jugement de valeur que l'on puisse porter sur une coalition commerciale ou industrielle, elle est, d’après sa nature même, une restriction à la concurrence et comme on l'a vu précédemment tout acte dont le but est de circonscrire indûment la concurrence est illégal en soi. Même l'argumentation proprement économique comme l'argument des prix raisonnables ou celui de la stabilisation des marchés n'a eu aucune valeur disculpatoire devant les cours de justice. ${ }^{3}$

Le bill C-59 présenté à la dernière session aborde cependant la question des monopoles sous un angle différent.

LE BILL C-59

Essentiellement, les changements que le bill C-59 aurait apportés à la législation anti-trust consistent dans l'énumération de pratiques commerciales qui ne pourraient être considérées illégales si le bill avait été voté par le Parlement. En d'autres mots, le bill C-59 maintient dans l'illégalité les ententes industrielles ou commerciales ayant pour objet la fixation des prix, la limitation de la production d'une marchandise, la division du marché entre les producteurs ou les distributeurs, la restriction de l'entrée dans l'industrie ainsi que toute autre pratique ayant pour but de restreindre les canaux de distribution; cependant, ce même

(1) Stinson-Reeb Co. et Al. V. The King, 1929.

(2) W.L. Mackenzie King, Débats de la Chambre des Communes, 1923, Vol. 3, pp. 2513 ss.

(3) cf. Marion, Gérald, La politique canadienne sur les monopoles, Inst. Soc. Popul., 1957, pp. 30 ss. 
bill classifie parmi les pratiques permises celles qui ont pour objet l'échange de statistiques, l'uniformisation des produits d'une industrie ou des conditions de commerce, la coopération dans la recherche ainsi que certaines autres pratiques que le législateur n'énumère pas. $\mathrm{De}$ plus, un autre paragraphe de la loi proposée permet à la défense d'utiliser comme argument disculpatoire le fait qu'une coalition n'agit pas au détriment de l'intérêt public. Enfin, une autre stipulation du bill permet la fusion d'entreprises commerciales ou industrielles lorsque cette intégration a pour effet de permettre la réalisation d'économies de dimension pourvu toutefois qu'un certain degré de concurrence demeure en dépit de la fusion. Pour terminer, un autre paragraphe de cet artiole permet les fusions si l'entreprise fusionnée peut démontrer que, ide toute façon, elle aurait cessé d'exister.

\section{LES COALITIONS}

Je veux dans les lignes qui vont suivre faire une analyse de certaines pratiques commerciales que la loi permet explicitement.

Les pratiques commerciales qui, selon le bill C-59, pourraient faire l'objet d'ententes ne sont pas des pratiques monopolistiques proprement dites, comme l'est, par exemple, la fixation directe du prix de vente d'un produit. Cependant, plusieurs de ces pratiques que le bill proposé permet sont des conditions préalables à l'exercice d'un pouvoir monopolistique, et en les permettant, le gouvernement pourrait rendre inefficace la lutte contre les monopoles. Je crois que c'est particulièrement le cas de 1) l'échange de statistiques, 2) l'uniformisation des produits et des conditions commerciales, 3) l'utilisation de la règle de raison dans l'évaluation des ententes.

D'après le bill C-59, les ententes commerciales ou industrielles pour la fixation des prix d'un produit sont illégales; pourtant, ce même bill permet l'échange de statistiques ou d'informations entre producteurs ou distributeurs d'une marchandise. On peut se demander si ces deux parties de la loi ne sont pas inconciliables en ce sens qu'une stipulation de la loi permet de contourner la loi elle-même: l'échange d'informations permise dans la loi rend parfois possible, à qui le désire, de prendre une attitude monopolistique sans avoir recours à l'entente directe qui est une pratique illégale. En effet, l'échange de statistiques entre entreprises est non seulement une condition nécessaire mais parfois suffisante pour permettre une entente tacite sur le prix de vente d'un produit; d'autre part, l'entente directe sur les prix est souvent un acte superflu. Voici comment. Il est un principe bien connu en économie politique qui veut que dans toute structure de marché oligopolistique, tout entrepreneur qui recherche un profit maximum tienne compte du comportement de ses rivaux et s'abstienne de les concurrencer chaque fois que le profit du groupe est plus élevé que si chacun agissait individuellement. Or la diffusion des informations que le bill C-59 rend explicitement légale permet justement aux entrepreneurs qui veu- 
lent porter leurs profits au point maximum de suivre des politiques de prix parallèles grâce à l'échange d'informations. En d'autres termes, ils pourront pratiquer le parallélisme conscient qui, comme on le sait, est une pratique monopolistique. En effet, les informations fournies par l'échange de statistiques permettront à un entrepreneur en particulier d'aligner sa politique de prix avec celle de ses rivaux chaque fois qu'il poussera son profit au point maximum de cette façon.

Pour illustrer davantage mon argumentation, je prends un autre cas, celui de la dominance des prix (price leadership) qui peut s'établir grâce à l'information sur l'état de l'offre d'un producteur en particulier. Supposons que dans une structure de marché où les producteurs sont peu nombreux, il appert, grâce aux statistiques échangées, que pour l'un d'entre eux, l'élasticité de l'offre est très élevée. Dans ce cas, ses concurrents éviteront, dans leur intérêt, de lui faire une guerre de prix et suivront ses politiques de prix.

On le voit, la diffusion des informations quant aux conditions de l'offre et de la demande peut pousser les entreprises à éviter la concurrence et permet le parallélisme conscient aussi bien que la dominance des prix. On sait d'autre part que ces pratiques ont les mêmes effets que les ententes directes, mais la preuve en est tellement difficile à faire que par le passé le gouvernement canadien a évité de les aborder.

La candeur avec laquelle le législateur a introduit cette stipulation dans la législation proposée pourrait laisser entendre qu'il s'agit de quelque chose de nouveau. Cependant, tel n'est pas le cas. L'un des premiers auteurs à préconiser l'échange de statistiques a été $\mathbf{A}$. J. Eddy, l'auteur de «New Competition ${ }^{*}$, selon qui la vraie concurrence n'existe que si les entreprises connaissent et peuvent juger les politiques des concurrents. Mais d'après l'exposé ci-haut, l'information ne conduit pas nécessairement au développement de la concurrence, au contraire. D'ailleurs, dans une cause célèbre entendue devant la Cour suprême des Etats-Unis, cette question d'échange d'informations dans ce contexte de la politique anti-trust a été longuement analysée et discutée. Il n'est pas superflu, ici, de donner un bref aperçu de cette cause importante. Il s'agit de American Column of Lumber Co. vs United States. ${ }^{5}$ Les faits sont les suivarits. Une association commerciale avait organisé un vaste réseau d'échange d'informations sur l'industrie du bois dans le but de renseigner de la façon la plus complète et la plus précise possible ses membres sur les conditions de l'offre et de la demande des produits de cette industrie. Les rapports envoyés à chaque membre étaient fréquents, très détaillés et incluaient entre autres la liste des prix demandés. Bien que les membres de cette

(4) EDDy, A.J., New Competition, Chicago, 1912.

(5) Supreme Court of the United States, 1921. (Il faut nuancer notre affirmation en disant que le département des statistiques de l'association laissait clairement voir qu'il était possible de hausser les prix, bien que toute entente formelle sur les prix fût exclue). 
association aient nié catégoriquement que leur but était de fixer les prix, l'honorable juge Clarke en a quand même déduit qu'une telle coopération était contraire à la libre concurrence. Nous avons là l'exemple qu'un réseau d'échange de statistiques bien organisé peut conduire, sinon à la suppression, du moins à la diminution de la concurrence. Le but de cet exposé sur l'échange de statistiques n'était pas de porter un jugement absolu sur l'opportunité de le défendre ou de le permettre, mais bien de montrer comment ces informations peuvent, à l'occasion, être utilisées pour restreindre la concurrence dans le commerce. Il s'est avéré dans le passé que certains instituts spécialisés, en fournissant des informations à leurs membres, ont rendu d'importants services. Cependant bien que toute affirmation en ce qui concerne ces réseaux d'information doive être nuancée et faire la part des choses, il demeure que toute tentative pour les faire servir comme base d̀ des «gentlemen's agreements 》 est une entorse à la concurrence oonsidérée comme le principe régulateur de notre économie.

Nous allons maintenant analyser les effets de l'uniformisation des produits sur la concurrence. Dans les modèles théoriques élaborés pour définir la concurrence parfaite, on a toujours soin de préciser que les produits doivent être homogènes; en d'autres mots, l'uniformisation des produits est une condition nécessaire à l'existence de la concurrence parfaite. Cependant, la politique économique ne peut pas être prisonnière d'une hypothèse logique; de plus, bien que l'utilisation de la concurrence parfaite comme moyen d'analyse rende d'excellents services, elle ne peut servir comme guide à l'action étant donné qu'elle est fondée sur un ensemble d'hypothèses restrictives.

Ce qui existe surtout, c'est un système caractérisé par la concurrence praticable; c'est-à-dire un système où la rivalité existe, mais où le mécanisme qui conduit à l'équilibre n'est pas conforme aux normes de la concurrence parfaite. Je veux mentionner entre autres les structures de marché oligopolistiques. Dans de tels cas, l'uniformisation des produits peut justement être la condition qui va permettre aux producteurs ou aux distributeurs d'un même produit de suivre une politique de prix identiques et d'éviter ainsi la concurrence. Il arrive, comme on peut le constater en lisant les rapports sur de prétendues coalitions publiées par le Ministère fédéral de la justice, que des coalitions ont tendance à se former dans les industries où il existe une capacité de production excédentaire. Dans ce cas, la concurrence est inutilement ruineuse pour les entrepreneurs parce qu'elle ne peut qu'entraîner une baisse des prix. Dans ce cas, si la capacité de production oisive est également distribuée entre les firmes de l'industrie, il existe une propension à l'entente. Cependant, plus le marché sur lequel ces firmes écoulent leurs produits est cinculaire, c'est-àdire plus le degré d'interdépendance entre les firmes est élevé, plus la propension à suivre une politique de prix identiques et à éviter la concurrence est élevée. Or, justement, l'uniformisation des produits élève le degré d'interdépendance entre les entreprises et les pousse 
ainsi à suivre une politique commune quant aux prix de leurs produits. Bien entendu, le bill anti-trust proposé à la dernière session fédérale proscrit les ententes horizontales, mais dans un contexte semblable à celui exposé ci-haut, la normalisation des produits ayant rendu le marché circulaire, les entrepreneurs sentiront instinctivement le besoin de suivre une politique de prix identiques et d'éviter la concurrence. Dans un tel contexte, la proscription des ententes horizontales n'est efficace que dans le cas où les producteurs sont nombreux, car dans une telle situation, pour pouvoir suivre des politiques communes, ils doivent au préalable réaliser un accord formel entre eux.

Le nouveau bill anti-trust aurait permis la coopération en ce qui concerne la recherche et les développements futurs. A l'heure actuelle certaines industries possèdent des instituts de recherche supportés par les entreprises qui en font partie. Dans certains cas, ces instituts consacrent une bonne part de leurs activités à la conservation des ressources. Il ne semble pas que da recherche, considérée sous cet angle, pose des problèmes en ce qui concerne la législation anti-trust.

Un point plus délicat concerne la recherche scientifique pour l'amélioration des processus de production ou des produits d'une industrie. Dans certains cas, ces recherches demandent une très forte concentration de capitaux et ne peuvent souvent se faire au niveau des firmes individuelles. Il faut une mise de fonds commune. Je ne crois pas qu'il soit réaliste de s'y objecter au nom de la sauvegarde de la concurrence, d'autant plus qu'en certains cas, cette manière de procéder peut stimuler la concurrence: c'est ce qui arrive dans une structure de marché caractérisée par une entreprise géante concurrencée par une kyrielle de petites firmes. Dans ce cas, la coopération entre les petites firmes sur le plan de recherche pourra les amener à développer des processus de production plus efficaces ou des produits plus attrayants, de sorte que ces petites entreprises pourront désormais concurrencer la firme dominante. Ainsi, la coopération dans la recherche scientifique place les petites firmes dans une meilleure situation concurrentielle vis-à-vis de l'entreprise dominante et peut conduire à une concurrence réelle au lieu du simulacre de rivalité qui existait auparavant.

Le point le plus important en ce qui concerne la coopération pour la recherche est l'octroi des brevets. Si le gouvernement permet la coopération dans la recherche scientifique, il faudra aussi qu'il ait une politique d'octroi de brevets très éclairée. En effet, la coopération dans la recherche pourra facilement conduire à la centralisation ou polarisation des brevets. Or, la centralisation des brevets n'est souvent qu'un genre particulier de coalition en vue d'éliminer la concurrence. C'est pourquoi, l'octroi de brevets devra être surveillé.

Sur la question des coalitions, un dernier point que je veux soulever concerne cette partie du bill proposé qui permet l'existence de coalition pourvu que les parties impliquées puissent démontrer qu'elles 
n'agissent pas au détriment de l'intérêt public. Cela signifie en somme que les ententes ne seraient plus condamnables en elles-mêmes mais qu'elles seraient permises pourvu que les intérêts du public ne soient pas lésés. Ainsi, on appliquerait dans l'évaluation des ententes la règle de raison au lieu de la règle per se: rejetant la condamnation des ententes a priori, les cours de justice s'embourberaient dans des évaluations comptables et autres pour savoir si les prix sont trop élevés; en outre, elles permettraient des enquêtes sur les structures de marché pour savoir si l'entrée est libre dans telle industrie, etc. On peut sérieusement se demander si une telle attitude de la part du législateur ne rendrait pas la législation anti-trust inopérante et inefficace, car une telle stipulation permettrait de contourner la loi elle-même? En effet, comme l'a dit le professeur Mason: «An attempt to push inquiry into effects very far is clearly an invitation to nonenforcement ${ }^{6}{ }^{6}$

De toute façon, l'utilisation de la règle de raison dans l'évaluation d'une pratique restrictive conduit à l'étude de questions extrêmement controversées auxquelles il n'existe pas de réponse définitive. Ainsi, ce serait le cas de toute tentative faite pour déterminer le caractère raisonnable du taux de profit dans une industrie.

D'un autre côté, on soutient que les coalitions n'ont pas seulement des implications d'ordre économique. Un système d'entreprise privée ne peut s'accommoder que d'un régime où le consommateur a pleine liberté de choix et où le pouvoir économique privé est exclu. ${ }^{\tau}$

Fusions

La législation proposée permet les fusions à certaines conditions. Une fusion est justifiée pourvu qu'elle permette la réalisation d'économies et qu'un certain degré de concurrence demeure dans l'industrie où elle a eu lieu.

La loi fait jusqu'à un certain point une distinction entre le contrôle du marché et l'efficacité économique. En permettant une fusion, la Cour permettrait à une industrie d'étendre son contrôle du marché pourvu que l'efficacité économique en soit améliorée. C'est la transposition, dans notre législation anti-trust, d’opinions exprimées par des économistes anglais, entre autres E. A. G. Robinson. ${ }^{8}$

Cependant, selon la lettre du bill, bien qu'une fusion soit justifiée par des économies de dimension ou d'échelle, il y aurait quand même une limite au contrôle du marché, à savoir qu'un certain degré de concurrence doit demeurer après que la fusion a eu lieu.

(6) Mason, E.S., Market Power and Business Conduct, American Economic Review, May 1956, p. 475.

(7) Wirson, G.W., Anti-combines and Injury to the Public, Canadian Joumal of Economics and Political Science, Feb. 1957, pp. 121-128.

(8) How Should We Control Monopoly? Economic Journal, Dec. 1956, pp. 569 ss. 
En considérant la question de l'expansion d'une firme par l'absorption de concurrents ainsi que la question de l'efficacité économique, on est d'abord amené à constater qu'il y a causalité réciproque entre les deux phénomènes. L'efficacité peut conduire à l'absorption de rivaux, tandis que l'expansion elle-même peut amener une plus grande efficacité par la création d'économies d'échelle.

Il semble que le degré d'efficacité influera sur le degré de tolérance que l'on aura pour la concentration économique résultant des fusions. Le contrôle du marché ne serait pas un critère absolu: on serait plus ou moins tolérant suivant que les entreprises fusionnées seraient plus ou moins efficaces.

Cela voudrait dire que l'on accepterait le passage du capitalisme atomistique au capitalisme de groupe pourvu que l'efficacité économique y gagne. On consentirait au nom de l'efficacité et du rendement économique à ce que des groupes particuliers détiennent des pouvoirs économiques élevés. Mais, comme l'ont affirmé quatre juges de la Cour suprême des Etats-Unis, de tels pouvoirs doivent être réservés aux représentants du peuple. «Le pouvoir de contrôler l'économie doit être entre les mains des élus du peuple, non entre les mains d'une oligarchie industrielle ${ }^{9}{ }^{9}$

Sans doute la loi dit que les économies qui ont été réalisées grâce à la fusion doivent passer au public; mais dans une économie dynamique, il est extrêmement difficile de faire une telle évaluation. Ainsi, en ce qui concerne les prix d'un produit, ceux qui sont considérés comme favorisant le public aujourd'hui peuvent, à la suite de changements économiques, devenir des prix excessifs. Pour savoir si le public bénéficie des économies réalisées grâce à une plus forte concentration, il faudrait sans cesse enquêter pour tenir compte des conditions microéconomiques et macro-économiques changeantes qui caractérisent l'économie contemporaine.

Enfin, le bill proposé stipule que toute fusion pourra être considérée comme légale si l'entreprise qui est fusionnée avait de toute façon cessé d'exister à cause de sa situation précaire. Pour discuter du bien-fondé de cette stipulation de la loi, il faut l'analyser dans deux hypothèses particulières. Dans une première situation, il arrive qu'après l'intégration, l'entreprise fusionnée, sans qu'un changement de structure ne soit opéré, redevienne économiquement rentable alors que préalablement à l'acquisition, elle était considérée comme inefficace. Dans ce cas, on peut se demander si les difficultés financières étaient réelles ou encore si cette non-rentabilité était provoquée par lartifice de la compagnie acquéreuse en vue de la transaction. Il est entendu que lorsque ces situations existent, l'argument de la non-rentabilité n'est qu'un prétexte utilisé pour contourner la loi.

(9) Douglas, Black, Murphy and Rutledge, United States vs Columbia Steel Co., Supreme Court of the United States, 1948 (décision minoritaire). 
Dans la deuxième hypothèse, la non-rentabilité est réelle. Il peut s'agir d'une entreprise dont l'équipement est désuet, sans aucune perspective d'amélioration. Dans ce cas, la fusion n'est pas nécessairement préjudiciable à l'intérêt public; elle peut éviter que des pertes autrement probables ne se produisent. Cependant, lorsqu'une entreprise se trouvant dans une telle situation est fusionnée, il est quand même important de considérer quelle sorte de structure de marché résultera de la consolidation. En effet, dans l'hypothèse où il existe plus d'un acquéreur possible, le résultat de la fusion quant à la ooncurrence pourra être différent selon que c'est l'un ou l'autre des acquéreurs potentiels qui de fait effectue la consolidation. Ainsi dans un marché caractérisé par l'existence d'une entreprise dominante et de quelques autres entreprises-satellites, la concurrence sera sauvegardée ou même améliorée si l'une des entreprises dominées se porte acquéreuse d'une autre firme également en situation de satellite sur le marché du produit. On le voit, le caractère du marché ainsi que la situation de la firme ont leur importance lorsqu'on considère les effets d'une consolidation.

\section{JURISPRUDENCE DU TRAVAIL}

\section{La procédure de grief à l'expiration de la covention collective}

Une convention collective étant expirée, larticle 24 de la Loi des relations ouvrières oblige l'employeur à respecter les conditions de travail, entre autres, les clauses de congédiement et d'ancienneté. La procédure des griefs est un accessoire pour faire valoir ces droits et elle continue à s'appliquer, même si le contrat est expiré.

Cause des griefs

Les causes de griefs sont les suivantes: dans la cause de Tousignant, il s'agit d'un renvoi ou congédiement. Dans la cause de Nolan et Lauzon, il s'agit de droits d'ancienneté ou de séniorité.

Les intéressés ont tenté d'établir tout d'abord une procédure de griefs, suivant les différents stades prévus à la convention. La Quemont Mining Corporation s'est refusée de participer à cette procédure et elle a clairement mani-

(1) Différend entre Quemont Mining Corporation et United Steelworkers of America, Local 4451, agent négociateur. Membres du tribunal: Honorable Camille Beaulieu, président; M. Roger Bédard, arbitre syndical; Me Marcel Cinq-Mars, arbitre patronal. 